\title{
GW23-e1471 CORRELATION BETWEEN CORONARY ARTERY LESION SEVERITY AND LONG-TERM CLINICAL OUTCOMES IN CHINESE HAN OCTOGENARIANS WITH ACUTE CORONARY SYNDROME
}

doi:10.1136/heartjnl-2012-302920k.28

Fu Zhenhong, Chen Yundai, Dong Wei, Chen Lian, Gai Luyue, Liu Hongbin, Sun Zhijun, Guo Jun, Ren Yihong, Xue Hao, Xue Hao. Department of Cardiology, Chinese People's Liberation Army General Hospital

Objectives There is little long-term outcome data regarding acute coronary syndrome (ACS) in Chinese Han octogenarians (>80 years old). Accordingly, we assessed the correlation between coronary artery lesion severity and long-term mortality in octogenarians with ACS.

Methods We classified 536 consecutive octogenarians with ACS based on the Gensini score into 4 groups: a control group (group 1), a group with Gensini score $<20$ (group 2), a group with Gensini score from 21 to 60 (group 3), and a group with Gensini score $>61$ (group 4). Survival and MACE rates were calculated using the Kaplan-Meier method. Multivariate Cox regression was used to identify mortality predictors.

Results There were 66 (12.3\%), 141 (26.3\%), 167 (31.2\%) and 162 $(30.2 \%)$ patients in groups $1,2,3$ and 4 , respectively. The average follow-up was $27.1 \pm 16.0$ months. Heart rate, systolic blood pressure (SBP), blood glucose level, e-GFR, morbidity from old myocardial infarction, smoking, ACS type, and GRACE score were the determinants of coronary artery lesion severity. Increasing coronary artery lesion severity was associated with increased long-term mortality and MACE rates. The overall long-term mortality rate was $9.1 \%$ and increased from $3.0 \%$ in group 1 to $16.7 \%$ in group 4 . Age, gender, heart rate, SBP, chronic renal failure, e-GFR, GRACE score, Gensini score, and ACS type were the independent predictors of long-term mortality.

Conclusions Long-term mortality of octogenarians with ACS was associated with increased coronary artery lesion severity. Gender and chronic renal failure were the major risk factors for long-term mortality. 